\title{
Rectangular Algebras as Tree Recognizers
}

\author{
Magnus Steinby*
}

To the memory of Ferenc Gécseg

\begin{abstract}
We consider finite rectangular algebras of finite type as tree recognizers. The type is represented by a ranked alphabet $\Sigma$. We determine the varieties of finite rectangular $\Sigma$-algebras and show that they form a Boolean lattice in which the atoms are minimal varieties of finite $\Sigma$-algebras consisting of projection algebras. We also describe the corresponding varieties of $\Sigma$-tree languages and compare them with some other varieties studied in the literature. Moreover, we establish the solidity properties of these varieties of finite algebras and tree languages. Rectangular algebras have been previously studied by R. Pöschel and M. Reichel (1993), and we make use of some of their results.
\end{abstract}

\section{Introduction}

In a projection algebra every fundamental operation is a projection operation. Pöschel and Reichel [11] defined rectangular $\tau$-algebras as the members of the variety generated by all projection algebras of type $\tau$. Rectangular algebras are also natural generalizations of rectangular bands; the rectangular algebras of type $\langle 2\rangle$ are precisely the rectangular bands.

In this paper we study projection algebras and rectangular algebras as tree recognizers. Hence the algebras considered are finite and of a finite type, represented here by a ranked alphabet $\Sigma$. Our general framework is the variety theory of tree languages [12,13], which establishes bijective correspondences between the varieties $\Sigma$-tree languages $(\Sigma$-VTLs), the varieties of finite $\Sigma$-algebras ( $\Sigma$-VFAs), and the $\Sigma$-varieties of finite congruences ( $\Sigma$-VFCs).

The class of all finite projection $\Sigma$-algebras is not a $\Sigma$-VFA, but it contains certain simple $\Sigma$-VFAs from which all the $\Sigma$-VFAs to be considered here are obtained. Each such atomic $\Sigma$-VFA corresponds to some so-called projection alphabet. For any projection alphabet $\Lambda$, the class $\mathbf{F P r o j}$ of all finite $\Lambda$-projection algebras is a minimal $\Sigma$-VFA, and these $\Sigma$-VFAs $\mathbf{F P r o j}_{\Lambda}$ are the atoms of the Boolean lattice of all sub-VFAs of the $\Sigma$-VFA $\mathbf{F R A _ { \Sigma }}$ of all finite rectangular $\Sigma$-algebras. Every sub-VFA FRA $\mathbf{A}_{\mathcal{L}}$ of $\mathbf{F R A}_{\Sigma}$ corresponds to a set $\mathcal{L}$ of projection alphabets, and it

\footnotetext{
*Department of Mathematics and Statistics, University of Turku, FIN-20014 Turku, Finland. E-mail: steinby@utu.fi
} 
is the finite join of the $\Sigma$-VFAs $\operatorname{FProj}_{\Lambda}$ such that $\Lambda \in \mathcal{L}$. We also describe the $\Sigma$-VFCs that correspond to the $\Sigma$-VFAs $\mathbf{F P r o j}_{\Lambda}$ and $\mathbf{F R A _ { \mathcal { L } }}$.

It is easy to describe a tree language recognized by a projection algebra; whether a tree is in it depends just on the label of the leaf at the end of a certain path determined by the projection alphabet of the algebra. This observation leads to a simple characterization of the members of the $\Sigma$-VTLs $F \operatorname{Proj}_{\Lambda}$ that correspond to the $\Sigma$-VFAs PProj $_{\Lambda}$. Moreover, we show that any tree language in $F \operatorname{Proj}_{\Lambda}$ is also recognized by a two-element $\Lambda$-projection algebra $\mathcal{P}_{\Lambda}$, which therefore is (up to isomorphism) the only nontrivial syntactic algebra in $\mathbf{F P r o j} \mathbf{j}_{\Lambda}$. We also note that the syntactic monoid of any member of $F \operatorname{Proj}_{\Lambda}$ is either trivial or isomorphic to a certain 3-element monoid. The $\Sigma$-VTLs $F R A_{\mathcal{L}}$ that correspond to the more general $\Sigma$-VFAs $\mathbf{F R} \mathbf{A}_{\mathcal{L}}$ are shown to be the ring closures of the unions of the atomic $\Sigma$-VTLs FProj $j_{\Lambda}$ they contain. It is also noted that the membership problem is decidable for these $\Sigma$-VTLs.

Although the tree languages recognized by rectangular algebras have rather simple descriptions, their trees are not characterized by any local properties. Therefore the $\Sigma$-VTLs $F R A_{\mathcal{L}}$ have little in common with many of the $\Sigma$-VTLs previously considered in the literature. Thus we show that the intersection of any $F R A_{\mathcal{L}}$ with any one of the $\Sigma$-VTLs of nilpotent, definite, reverse definite, generalized definite or locally testable $\Sigma$-tree languages is just the trivial $\Sigma$-VTL. Of course, the corresponding facts hold for $\Sigma$-VFAs. On the other hand, we show that $F R A_{\Sigma}$ is contained in the $\Sigma$-VFA of all aperiodic $\Sigma$-tree languages. As another exception, we show that for any projection alphabet $\Lambda$, the $\Sigma$-VTL $F \operatorname{Proj}_{\Lambda}$ is contained in the family $D R e c_{\Sigma}$ of $\Sigma$-tree languages recognized by deterministic top-down tree recognizers. This implies that $F R A_{\Sigma}$ is contained in the $\Sigma$-VTL generated by DRecs.

We also study the solidity properties of our $\Sigma$-VFAs and $\Sigma$-VTLs. Graczyńska and Schweigert [7] noted that the solidity of a class of algebras can be defined in terms of derived algebras. A derived algebra $\varkappa(\mathcal{A})$ of a $\Sigma$-algebra $\mathcal{A}$ is obtained by replacing each fundamental operation of $\mathcal{A}$ with a term operation determined by the given hypersubstitution $\varkappa$, and a class $\mathbf{K}$ of $\Sigma$-algebras is solid if it contains all derived algebras of its members. A family of $\Sigma$-tree languages is said to be solid, if it is closed under inverse tree homomorphisms. In fact, we consider the more refined notions of solidity with respect to a given class of hypersubstitutions. In [11] it was shown that the rectangular $\Sigma$-algebras form the least nontrivial solid variety of $\Sigma$-algebras, and hence it is to be expected that $\mathbf{F R A} \mathbf{A}_{\Sigma}$ is the least nontrivial solid $\Sigma$ VFA. Also the $\Sigma$-VFA of trivial $\Sigma$-algebras is naturally solid, but the remaining subVFAs of $\mathbf{F R A _ { \Sigma }}$ are shown to have very weak solidity properties. The corresponding facts hold for the $\Sigma$-VTLs $F R A_{\mathcal{L}}$.

\section{Preliminaries}

We may write $A:=B$ to emphasize that $A$ is defined to be $B$. For any integer $n \geq 0$, let $[n]:=\{1, \ldots, n\}$. The set of all subsets of a set $A$ is denoted by $\wp(A)$. 
For any relation $\rho \subseteq A \times B$, the fact that $(a, b) \in \rho$ for some $a \in A$ and $b \in B$, will usually be expressed by writing $a \rho b$. For a mapping $\varphi: A \rightarrow B$, we may write the image $\varphi(a)$ of an element $a \in A$ as $a \varphi$. Especially homomorphisms are written this way as right operators that are composed from left to right, i.e., the composition of $\varphi: A \rightarrow B$ and $\psi: B \rightarrow C$ is written as $\varphi \psi$.

Next we recall some basic matters concerning algebras, tree recognizers and tree languages. For details and further references, cf. [1, 5, 6, 13], for example.

A ranked alphabet $\Sigma$ is a finite set of symbols each of which has a unique positive integer arity. For any $m \geq 1$, the set of $m$-ary symbols in $\Sigma$ is denoted by $\Sigma_{m}$. Note that we assume that there are no nullary symbols. If $\Sigma=\Sigma_{1}$, then $\Sigma$ is said to be unary. The rank type of $\Sigma$ is the set $r(\Sigma):=\left\{m \mid \Sigma_{m} \neq \emptyset\right\}$. The ranked alphabet $\Sigma$ will have two roles. Firstly, the inner nodes of trees are labeled with symbols from $\Sigma$. Secondly, $\Sigma$ is a finite set of operation symbols that determines the type of the algebras to be considered. To avoid exceptions for the unary case, we make the following general assumption.

Convention. From now on, $\Sigma$ is a ranked alphabet without nullary symbols that contains at least one symbol of arity $\geq 2$.

We also use ordinary finite nonempty alphabets $X, Y, \ldots$ that we call leaf alphabets. These are assumed to be disjoint from $\Sigma$. For any leaf alphabet $X$, the set $T_{\Sigma}(X)$ of $\Sigma$-terms over $X$ is the smallest set $T$ such that $X \subseteq T$, and $f\left(t_{1}, \ldots, t_{m}\right) \in T$ whenever $m \in r(\Sigma), f \in \Sigma_{m}$ and $t_{1}, \ldots, t_{m} \in T$. Such terms are regarded in the usual way as labeled trees, and we call them $\Sigma X$-trees. Subsets of $T_{\Sigma}(X)$ are called $\Sigma X$-tree languages. We may also speak about $\Sigma$-trees and $\Sigma$-tree languages without specifying the leaf alphabet, or just about trees and tree languages. A family of $\Sigma$-tree languages is a mapping $\mathcal{V}$ that assigns to every leaf alphabet $X$ a set $\mathcal{V}(X)$ of $\Sigma X$-tree languages. We write such a family as $\mathcal{V}=\{\mathcal{V}(X)\}_{X}$. For any two such families $\mathcal{U}$ and $\mathcal{V}$, we set $\mathcal{U} \subseteq \mathcal{V}$ iff $\mathcal{U}(X) \subseteq \mathcal{V}(X)$ for every $X$. Unions and intersections of families of $\Sigma$-tree languages are defined by similar componentwise conditions.

Let $\xi$ be a special symbol that does not appear in $\Sigma$ or $X . \mathrm{A} \Sigma(X \cup\{\xi\})$-tree in which $\xi$ appears exactly once, is called a $\Sigma X$-context. The set of all $\Sigma X$-contexts is denoted by $C_{\Sigma}(X)$. If $p, q \in C_{\Sigma}(X)$ and $t \in T_{\Sigma}(X)$, then $p \cdot q=q(p)$ and $t \cdot q=q(t)$ are the $\Sigma X$-context and the $\Sigma X$-tree obtained from $q$ by replacing the $\xi$ in it with $p$ or $t$, respectively. Clearly, $C_{\Sigma}(X)$ forms a monoid for the product $p \cdot q$ and the identity element $\xi$.

A $\Sigma$-algebra $\mathcal{A}$ consists of a nonempty set $A$ and a $\Sigma$-indexed family $\left(f^{\mathcal{A}} \mid f \in \Sigma\right)$ such that if $f \in \Sigma_{m}$, then $f^{\mathcal{A}}: A^{m} \rightarrow A$ is an $m$-ary operation on $A$. We write simply $\mathcal{A}=(A, \Sigma)$. Subalgebras, homomorphisms, (epimorphic) images and direct products are defined as usual. An algebra $\mathcal{B}$ is said to cover an algebra $\mathcal{A}$ if $\mathcal{A}$ is an image of a subalgebra of $\mathcal{B}$. This we express by writing $\mathcal{A} \preceq \mathcal{B}$. The $\Sigma X$ trees form the $\Sigma X$-term algebra $\mathcal{T}_{\Sigma}(X)=\left(T_{\Sigma}(X), \Sigma\right)$, where $f^{\mathcal{T}_{\Sigma}(X)}\left(t_{1}, \ldots, t_{m}\right)=$ $f\left(t_{1}, \ldots, t_{m}\right)$ for all $m \in r(\Sigma), f \in \Sigma_{m}$ and $t_{1}, \ldots, t_{m} \in T_{\Sigma}(X)$.

A (deterministic bottom-up) $\Sigma X$-recognizer $\mathbf{A}=(\mathcal{A}, \alpha, F)$ consists of a finite $\Sigma$-algebra $\mathcal{A}=(A, \Sigma)$, the elements of which are called states, an initial assignment 
$\alpha: X \rightarrow A$ that specifies the starting states at the leaves, and a set $F \subseteq A$ of final states. The root of a $\Sigma X$-tree $t$ is reached in state $t \alpha_{\mathcal{A}}$, where $\alpha_{\mathcal{A}}: \mathcal{T}_{\Sigma}(X) \rightarrow \mathcal{A}$ is the homomorphic extension of $\alpha$, and hence the $\Sigma X$-tree language recognized by $\mathbf{A}$ is defined as $T(\mathbf{A})=\left\{t \in T_{\Sigma}(X) \mid t \alpha_{\mathcal{A}} \in F\right\}$.

A $\Sigma X$-tree language is called recognizable, or regular, if it is recognized by a $\Sigma X$-recognizer. Let $\operatorname{Rec}_{\Sigma}(X)$ be the set of all recognizable $\Sigma X$-tree languages, and let $\operatorname{Rec}_{\Sigma}=\left\{\operatorname{Rec}_{\Sigma}(X)\right\}_{X}$ be the family of recognizable $\Sigma$-tree languages. We may also say that a $\Sigma$-algebra $\mathcal{A}=(A, \Sigma)$ recognizes a $\Sigma X$-tree language $T$ if $T=F \varphi^{-1}$ for some homomorphism $\varphi: \mathcal{T}_{\Sigma}(X) \rightarrow \mathcal{A}$ and some $F \subseteq A$. Obviously, a $\Sigma X$-tree language is recognized by a finite algebra iff it is regular.

The following review of the variety theory of tree languages follows [12] and [13], where also further references can be found. The syntactic algebra of a $\Sigma X$-tree language $T$ is the quotient algebra $\operatorname{SA}(T):=\mathcal{T}_{\Sigma}(X) / \theta_{T}$, where $\theta_{T}$ is the syntactic congruence of $T$ defined by

$$
s \theta_{T} t \Leftrightarrow\left(\forall p \in C_{\Sigma}(X)\right)(p(s) \in T \leftrightarrow p(t) \in T) \quad\left(s, t \in T_{\Sigma}(X)\right) .
$$

It is easy to see that $\mathrm{SA}(T)$ is the minimal $\Sigma$-algebra recognizing $T$ in the sense that a $\Sigma$-algebra $\mathcal{A}$ recognizes $T$ iff $\operatorname{SA}(T) \preceq \mathcal{A}$.

A variety of $\Sigma$-tree languages $(\Sigma$-VTL) is a family of $\Sigma$-tree languages $\mathcal{V}=$ $\{\mathcal{V}(X)\}_{X}$ such that for all leaf alphabets $X$ and $Y$,

$(\mathrm{V} 1) \mathcal{V}(X)$ is a Boolean subalgebra of $\operatorname{Rec}_{\Sigma}(X)$,

(V2) if $T \in \mathcal{V}(X)$ and $p \in C_{\Sigma}(X)$, then $p^{-1}(T):=\left\{t \in T_{\Sigma}(X) \mid p(t) \in T\right\} \in \mathcal{V}(X)$, and

(V3) if $T \in \mathcal{V}(Y)$, then $T \varphi^{-1}:=\left\{t \in T_{\Sigma}(X) \mid t \varphi \in T\right\}$ is in $\mathcal{V}(X)$ for every homomorphism $\varphi: \mathcal{T}_{\Sigma}(X) \rightarrow \mathcal{T}_{\Sigma}(Y)$.

The least $\Sigma$-VTL is $\operatorname{Triv}_{\Sigma}=\left\{\operatorname{Triv}_{\Sigma}(X)\right\}_{X}$, where $\operatorname{Triv}_{\Sigma}(X)=\left\{\emptyset, T_{\Sigma}(X)\right\}$, and the greatest $\Sigma$-VTL is $\operatorname{Rec}_{\Sigma}=\left\{\operatorname{Rec}_{\Sigma}(X)\right\}_{X}$.

A class of finite $\Sigma$-algebras $\mathbf{K}$ is called a variety of finite $\Sigma$-algebras ( $\Sigma$-VFA) (or a pseudovariety) if it is closed under subalgebras, epimorphic images and finite direct products, i.e., if $S(\mathbf{K}), H(\mathbf{K}), P_{f}(\mathbf{K}) \subseteq \mathbf{K}$. The $\Sigma$-VFA generated by a class $\mathbf{K}$ of finite $\Sigma$-algebras is denoted by $V_{f}(\mathbf{K})$. Since $V_{f}(\mathbf{K})=H S P_{f}(\mathbf{K})$, a $\Sigma$-algebra $\mathcal{A}$ is in $V_{f}(\mathbf{K})$ iff $\mathcal{A} \preceq \mathcal{A}_{1} \times \ldots \times \mathcal{A}_{n}$ for some $n \geq 0$ and algebras $\mathcal{A}_{1}, \ldots, \mathcal{A}_{n} \in \mathbf{K}$. Let $\operatorname{Triv}_{\Sigma}$ be the $\Sigma$-VFA of all trivial $\Sigma$-algebras.

For any $\Sigma$ and $X$, let $\mathrm{FC}_{\Sigma}(X):=\left\{\theta \in \operatorname{Con}\left(\mathcal{T}_{\Sigma}(X)\right) \mid T_{\Sigma}(X) / \theta\right.$ finite $\}$ be the set of finite congruences of $\mathcal{T}_{\Sigma}(X)$. If $\Gamma$ assigns to each leaf alphabet a subset $\Gamma(X)$ of $\mathrm{FC}_{\Sigma}(X)$, we write $\Gamma=\{\Gamma(X)\}_{X}$, and we call $\Gamma$ a $\Sigma$-variety of finite congruences $(\Sigma$-VFC) if for all $X$ and $Y$,

(C1) $\Gamma(X)$ is a filter of the lattice $\left(\mathrm{FC}_{\Sigma}(X), \subseteq\right)$, and

(C2) $\varphi \circ \theta \circ \varphi^{-1}:=\left\{(s, t) \mid s, t \in T_{\Sigma}(X), s \varphi \theta t \varphi\right\}$ belongs to $\Gamma(X)$ for every $\theta \in \Gamma(Y)$ and every homomorphism $\varphi: \mathcal{T}_{\Sigma}(X) \rightarrow \mathcal{T}_{\Sigma}(Y)$. 
The three classes of varieties defined above form complete lattices with respect to the natural inclusion relations. They are connected by three pairs of mutually inverse isomorphisms.

For any $\Sigma$-VFA $\mathbf{K}$, let $\mathbf{K}^{t}$ be the family of $\Sigma$-tree languages and let $\mathbf{K}^{c}$ be the $\Sigma$-family of finite congruences such that for each $X, \mathbf{K}^{t}(X)=\left\{T \subseteq T_{\Sigma}(X) \mid\right.$ $\mathrm{SA}(T) \in \mathbf{K}\}$ and $\mathbf{K}^{c}(X)=\left\{\theta \in \mathrm{FC}_{\Sigma}(X) \mid \mathcal{T}_{\Sigma}(X) / \theta \in \mathbf{K}\right\}$. For any $\Sigma$-VTL $\mathcal{V}=\{\mathcal{V}(X)\}_{X}$, let $\mathcal{V}^{a}$ be the $\Sigma$-VFA generated by the syntactic algebras of the tree languages belonging to $\mathcal{V}$, and let $\mathcal{V}^{c}$ be the $\Sigma$-family of finite congruences such that for any $X, \mathcal{V}^{c}(X):=\left[\left\{\theta_{T} \mid T \in \mathcal{V}(\Sigma, X)\right\}\right)$ is the filter of $\mathrm{FC}_{\Sigma}(X)$ generated by the syntactic congruences of the members of $\mathcal{V}(X)$. Finally, for any $\Sigma$-VFC $\Gamma=\{\Gamma(X)\}_{X}$, let $\Gamma^{a}:=V_{f}\left(\left\{\mathcal{T}_{\Sigma}(X) / \theta \mid \theta \in \Gamma(X)\right.\right.$ for some $\left.\left.X\right\}\right)$ and let $\Gamma^{t}$ be the family of $\Sigma$-tree languages such that for any $X, \Gamma^{t}(X)=\left\{T \subseteq T_{\Sigma}(X) \mid \theta_{T} \in \Gamma(X)\right\}$. The Variety Theorem for $\Sigma$-tree languages can now be stated as follows.

Theorem 2.1. The mappings $\mathbf{K} \mapsto \mathbf{K}^{t}, \mathcal{V} \mapsto \mathcal{V}^{a}, \mathbf{K} \mapsto \mathbf{K}^{c}, \Gamma \mapsto \Gamma^{a}, \mathcal{V} \mapsto \mathcal{V}^{c}$, and $\Gamma \mapsto \Gamma^{t}$ form three pairs of mutually inverse isomorphisms between the lattices of all $\Sigma-V F A s, \Sigma-V T L s$ and $\Sigma-V F C s$.

A $\Sigma$-VFC $\Gamma=\{\Gamma(X)\}_{X}$ is principal if for every $X, \Gamma(X)$ is a principal filter in $\mathrm{FC}_{\Sigma}(X)$. It is easy to see that a family $\Gamma=\left\{\left[\gamma_{X}\right)\right\}_{X}$, where $\gamma_{X} \in \mathrm{FC}_{\Sigma}(X)$ for each $X$, is a principal $\Sigma$-VFC iff for all $X$ and $Y, \gamma_{X} \subseteq \varphi \circ \gamma_{Y} \circ \varphi^{-1}$ for every homomorphism $\varphi: \mathcal{T}_{\Sigma}(X) \rightarrow \mathcal{T}_{\Sigma}(Y)$.

Remark 2.1. If $\Gamma=\left\{\left[\gamma_{X}\right)\right\}_{X}$ is a principal $\Sigma$-VFC, then $\Gamma^{t}(X)$ is the finite set of $\Sigma X$-tree languages saturated by $\gamma_{X}$. Conversely, if $\mathcal{V}=\{\mathcal{V}(X)\}_{X}$ is a $\Sigma$-VTL such that $\mathcal{V}(X)$ is a finite set for every $X$, then $\mathcal{V}^{c}$ is a principal $\Sigma$-VFC because the filter $\mathcal{V}^{c}(X)$ is generated by the syntactic congruences of the members of $\mathcal{V}(X)$.

The join of any finite set of $\Sigma$-VFAs can be described as follows.

Lemma 2.1. For any $\Sigma$-VFAs $\mathbf{K}_{1}, \ldots, \mathbf{K}_{n}(n \geq 1)$, the join $\mathbf{K}_{1} \vee \ldots \vee \mathbf{K}_{n}=$ $V_{f}\left(\mathbf{K}_{1} \cup \ldots \cup \mathbf{K}_{n}\right)$ consists of all $\Sigma$-algebras $\mathcal{A}$ such that $\mathcal{A} \preceq \mathcal{A}_{1} \times \ldots \times \mathcal{A}_{n}$ for some $\mathcal{A}_{1} \in \mathbf{K}_{1}, \ldots, \mathcal{A}_{n} \in \mathbf{K}_{n}$.

The $\Sigma$-VTL generated by a family of recognizable $\Sigma$-tree languages $\mathcal{V}$ is the least $\Sigma$-VTL containing $\mathcal{V}$. The Boolean closure $\mathfrak{B} \mathcal{V}$ and the ring closure $\mathfrak{R} \mathcal{V}$ of $\mathcal{V}$ are the families of $\Sigma$-tree languages such that for any $X, \mathfrak{B} \mathcal{V}(X)$ is the Boolean closure of $\mathcal{V}(X)$ in $\operatorname{Rec}_{\Sigma}(X)$ and $\mathfrak{R} \mathcal{V}(X)$ is the least subset of $\operatorname{Rec}_{\Sigma}(X)$ containing $\mathcal{V}(X)$ and closed under finite intersections and unions.

Lemma 2.2. If a family of recognizable $\Sigma$-tree languages $\mathcal{V}=\{\mathcal{V}(X)\}_{X}$ satisfies conditions (V2) and (V3), then $\mathfrak{B} \mathcal{V}$ is the $\Sigma$-VTL generated by $\mathcal{V}$. If, moreover, $T \in \mathcal{V}(X)$ implies $T^{\complement} \in \mathcal{V}(X)$ for every $X$, then $\mathfrak{R} \mathcal{V}$ is the $\Sigma$-VTL generated by $\mathcal{V}$.

Proof. The lemma follows from the identities $p^{-1}\left(T \cup T^{\prime}\right)=p^{-1}(T) \cup p^{-1}\left(T^{\prime}\right)$, $p^{-1}\left(T^{\complement}\right)=p^{-1}(T)^{\complement},\left(T \cup T^{\prime}\right) \varphi^{-1}=T \varphi^{-1} \cup T^{\prime} \varphi^{-1}$ and $T^{\complement} \varphi^{-1}=\left(T \varphi^{-1}\right)^{\complement}$, where $p$ and $\varphi$ are as in (V2) and (V3) and $T$ and $T^{\prime}$ are tree languages of the appropriate kind. 
The join $\mathcal{V}_{1} \vee \ldots \vee \mathcal{V}_{n}$ of any $\Sigma$-VTLs $\mathcal{V}_{1}=\left\{\mathcal{V}_{1}(X)\right\}_{X}, \ldots, \mathcal{V}_{n}=\left\{\mathcal{V}_{n}(X)\right\}_{X}$ $(n \geq 1)$ is naturally the $\Sigma$-VTL generated by the union $\mathcal{V}_{1} \cup \ldots \cup \mathcal{V}_{n}=\left\{\mathcal{V}_{1}(X) \cup\right.$ $\left.\ldots \cup \mathcal{V}_{n}(X)\right\}_{X}$. Since the $\mathcal{V}_{i}$ s are $\Sigma$-VTLs, this union satisfies the conditions of Lemma 2.2, and we get

Corollary 2.1. If $\mathcal{V}_{1}, \ldots, \mathcal{V}_{n}(n \geq 1)$ are $\Sigma$-VTLs, then $\mathcal{V}_{1} \vee \ldots \vee \mathcal{V}_{n}=\mathfrak{R}\left(\mathcal{V}_{1} \cup\right.$ $\left.\ldots \cup \mathcal{V}_{n}\right)$.

\section{$3 \quad$ Projection algebras and rectangular algebras}

For any $m>0$ and $i \in[m]$, the $i^{\text {th }}$ m-ary projection operation on a set $A$ is the mapping $e_{i}^{m}: A^{m} \rightarrow A,\left(a_{1}, \ldots, a_{m}\right) \mapsto a_{i}$. (We omit $A$ from the notation as it is always known from the context.) An algebra $\mathcal{A}=(A, \Sigma)$ is called [11] a projection algebra if for all $m \in r(\Sigma)$ and $f \in \Sigma_{m}$, there is an $i \in[m]$ for which $f^{\mathcal{A}}=e_{i}^{m}$. Let $\mathbf{F P r o j}_{\Sigma}$ denote the class of all finite projection $\Sigma$-algebras. The direct product of projection algebras is in general not a projection algebra, but we shall show that $\mathbf{F P r o j}_{\Sigma}$ contains subclasses that are $\Sigma$-VFAs.

The path alphabet of $\Sigma$ is the set $\widehat{\Sigma}:=\bigcup\left\{\Sigma_{m} \times[m] \mid m \in r(\Sigma)\right\}$ regarded as an ordinary alphabet. We shall write $f_{i}$ for $(f, i)$. Words over $\widehat{\Sigma}$ describe paths in trees; if $f_{i}$ appears in such a word, then $f$ labels a node on the path and $i$ indicates the direction taken at that node. We call a subalphabet $\Lambda$ of $\widehat{\Sigma}$ a projection alphabet if for all $m \in r(\Sigma)$ and $f \in \Sigma_{m}$, there is exactly one $i \in[m]$ such that $f_{i} \in \Lambda$. Let $p a(\Sigma)$ denote the set of all projection alphabets over $\Sigma$. If $\Lambda \in p a(\Sigma)$, the $\Lambda$-path $\Lambda(t)$ in a $\Sigma X$-tree $t$ is defined as follows:

(1) $\Lambda(x)=x$ for every $x \in X$;

(2) $\Lambda(t)=f_{i} \Lambda\left(t_{i}\right)$ if $t=f\left(t_{1}, \ldots, t_{m}\right)$ and $f_{i} \in \Lambda$.

Obviously, $\Lambda(t)$ is always of the form $w x$, where $w \in \Lambda^{*}$ and $x \in X$. The word $w$ describes a path from the root to a leaf and $x$ is the label of that leaf. Let $\Lambda^{\bullet}(t)$ denote this label $x$. Each projection algebra $\mathcal{A}$ defines a projection alphabet

$$
\Lambda_{\mathcal{A}}:=\left\{f_{i} \mid f \in \Sigma_{m}, m \in r(\Sigma), i \in[m], f^{\mathcal{A}}=e_{i}^{m}\right\}
$$

and conversely, given the set $A$, this projection alphabet determines the projection algebra $\mathcal{A}$.

Definition 3.1. For any $\Lambda \in p a(\Sigma)$, we call a projection algebra $\mathcal{A}=(A, \Sigma)$ a $\Lambda$-projection algebra if $\Lambda_{\mathcal{A}}=\Lambda$. The class of all finite $\Lambda$-projection algebras is denoted by $\mathbf{F P r o j}_{\Lambda}$.

Let $\mathcal{A}$ and $\mathcal{B}$ be projection algebras. It is clear that if $\mathcal{A}$ is a subalgebra or an image of $\mathcal{B}$, then $\Lambda_{\mathcal{A}}=\Lambda_{\mathcal{B}}$. Moreover, it is easy to see that for any projection alphabet $\Lambda \in p a(\Sigma)$ the direct product of any family of $\Lambda$-projection algebras is a $\Lambda$-projection algebra. Hence, any $\mathbf{F P r o j}_{\Lambda}$ is a $\Sigma$-VFA. However, we can say a bit more about these classes. 
For each $\Lambda \in p a(\Sigma)$, let $\mathcal{P}_{\Lambda}=(\{0,1\}, \Sigma)$ be the two-element $\Lambda$-projection algebra. In [11] it was shown that the two-element projection algebras are the only nontrivial subdirectly irreducible projection algebras. Hence the following proposition is obvious.

Proposition 3.1. For any projection alphabet $\Lambda \in p a(\Sigma), \mathcal{P}_{\Lambda}$ is the only nontrivial subdirectly irreducible algebra in $\mathbf{F P r o j _ { \Lambda }}$, and hence every algebra in $\mathbf{F P r o \mathbf { j } _ { \Lambda }}$ is a finite subdirect power of $\mathcal{P}_{\Lambda}$. Moreover, $\mathbf{F P r o j}_{\Lambda}$ is a minimal $\Sigma$-VFA.

For any $\Lambda \in p a(\Sigma)$ and any leaf alphabet $X$, let

$$
\rho_{\Lambda}(X):=\left\{(s, t) \mid s, t \in T_{\Sigma}(X), \Lambda^{\bullet}(s)=\Lambda^{\bullet}(t)\right\}
$$

It is easy to see that $\rho_{\Lambda}(X)$ is a congruence on $\mathcal{T}_{\Sigma}(X)$ and that the $\rho_{\Lambda}(X)$-classes are precisely the sets $[x]:=\left\{t \in T_{\Sigma}(X) \mid \Lambda^{\bullet}(t)=x\right\}$, where $x \in X$. Hence the quotient algebra $\mathcal{F}_{\Lambda}(X):=\mathcal{T}_{\Sigma}(X) / \rho_{\Lambda}(X)$ has $|X|$ elements. Moreover, for any $m \in r(\Sigma), f \in \Sigma_{m}$ and $x_{1}, \ldots, x_{m} \in X$,

$$
f^{\mathcal{F}_{\Lambda}(X)}\left(\left[x_{1}\right], \ldots,\left[x_{m}\right]\right)=\left[x_{i}\right]
$$

for the $i \in[m]$ such that $f_{i} \in \Lambda$. Furthermore, it is clear that if $\mathcal{A}=(A, \Sigma)$ is a $\Lambda$-projection algebra, then any mapping $\varphi:\{[x] \mid x \in X\} \rightarrow A$ is a homomorphism from $\mathcal{F}_{\Lambda}(X)$ to $\mathcal{A}$. Hence, $\mathcal{F}_{\Lambda}(X)$ is freely generated over the class of all $\Lambda$-projection algebras by the set $\{[x] \mid x \in X\}$. Since $\mathcal{F}_{\Lambda}(X)$ is finite, it belongs to $\mathbf{F P r o j}_{\Lambda}$, and therefore $\rho_{\Lambda}(X)$ is the least congruence $\theta$ on $\mathcal{T}_{\Sigma}(X)$ such that $\mathcal{T}_{\Sigma}(X) / \theta \in \mathbf{F P r o j}_{\Lambda}$. This means that $\mathbf{F P r o j}_{\Lambda}^{c}$ is the principal $\Sigma$-VFC $\left\{\left[\rho_{\Lambda}(X)\right)\right\}_{X}$. These observations are summarized by the following proposition.

Proposition 3.2. For any $\Lambda \in p a(\Sigma)$ and any $X$, the set $\{[x] \mid x \in X\}$ generates $\mathcal{F}_{\Lambda}(X)$ freely over $\mathbf{F P r o j}_{\Lambda}$. Moreover, $\mathbf{F P r o j}_{\Lambda}^{c}=\left\{\left[\rho_{\Lambda}(X)\right)\right\}_{X}$.

The variety generated by all projection algebras of a given, not necessarily finite, type was studied by Pöschel and Reichel [11] who called its members rectangular algebras. Let us note that the rectangular algebras appear also in Ésik [3] in the form of "diagonal theories". We denote by $\mathbf{R} \mathbf{A}_{\Sigma}$ the variety of rectangular $\Sigma$ algebras and by $\mathbf{F R A}_{\Sigma}$ the $\Sigma$-VFA formed by the finite rectangular $\Sigma$-algebras. Let us now exhibit all the sub-VFAs of $\mathbf{F R A}_{\Sigma}$.

For any set $\mathcal{L} \subseteq \wp(\widehat{\Sigma})$ of projection alphabets, let $\mathbf{F R} \mathbf{A}_{\mathcal{L}}$ denote the join $V_{f}\left(\bigcup_{\Lambda \in \mathcal{L}} \mathbf{F P r o j}_{\Lambda}\right)$ of the $\Sigma$-VFAs FProj $_{\Lambda}$ with $\Lambda \in \mathcal{L}$. Of course, FRA $_{p a(\Sigma)}=$ $\mathbf{F R A}_{\Sigma}$ and $\mathbf{F R A}_{\{\Lambda\}}=\mathbf{F P r o j}_{\Lambda}$ for each $\Lambda \in p a(\Sigma)$. The nontrivial subdirectly irreducible members of $\mathbf{F R A _ { \mathcal { L } }}$ are the algebras $\mathcal{P}_{\Lambda}=(\{0,1\}, \Sigma)$ with $\Lambda \in \mathcal{L}$, and

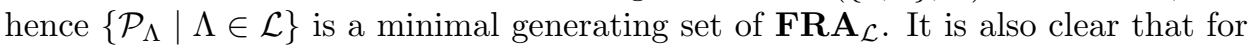
any $\mathcal{L}, \mathcal{M} \subseteq p a(\Sigma)$,

(1) FRA $_{\mathcal{L}} \subset$ FRA $_{\mathcal{M}}$ iff $\mathcal{L} \subset \mathcal{M}$, and

(2) FRA $_{\mathcal{L}} \cap \mathbf{F R A}_{\mathcal{M}}=\operatorname{Triv}_{\Sigma}$ if $\mathcal{L} \cap \mathcal{M}=\emptyset$. 
Let $n(\Sigma)$ denote the product of the arities of the symbols in $\Sigma$. It is clear that $n(\Sigma)$ is the number of projection alphabets $\Lambda \in p a(\Sigma)$, and therefore also the number the algebras $\mathcal{P}_{\Lambda}$. As noted in [11], this means that $\mathbf{R} \mathbf{A}_{\Sigma}$ has precisely $2^{n(\Sigma)}$ subvarieties. Using the above observations, we can formulate the corresponding statement for $\mathbf{F R A _ { \Sigma }}$ in the following more detailed form.

Proposition 3.3. The $\Sigma$-VFAs $\mathbf{F R A}_{\mathcal{L}}(\mathcal{L} \subseteq$ pa $(\Sigma))$ form a $2^{n(\Sigma)}$-element Boolean sublattice of the lattice of all $\Sigma$-VFAs. In this sublattice

(1) the least element is $\operatorname{Triv}_{\Sigma}$, the greatest element is $\mathbf{F R A _ { \Sigma }}$,

(2) $\mathbf{F R A}_{\mathcal{L}} \vee \mathbf{F R A}_{\mathcal{M}}=\mathbf{F R A}_{\mathcal{L} \cup \mathcal{M}}, \mathbf{F R A}_{\mathcal{L}} \wedge \mathbf{F R A}_{\mathcal{M}}=\mathbf{F R A}_{\mathcal{L} \cap \mathcal{M}}$, and $\mathbf{F R A}_{\mathcal{L}}^{\complement}=$ FRA $_{p a(\Sigma) \backslash \mathcal{L}}$, for all $\mathcal{L}, \mathcal{M} \subseteq p a(\Sigma)$, and

(3) the atoms are the minimal $\Sigma-V F A s \mathbf{F P r o j}_{\Lambda}$ with $\Lambda \in p a(\Sigma)$.

The join $\mathbf{F R A}_{\mathcal{L}}^{c}$ of the $\Sigma$-VFCs $\mathbf{F P r o j}_{\Lambda}^{c}$ with $\Lambda \in \mathcal{L}$, is the principal $\Sigma$-VFC $\left\{\left[\rho_{\mathcal{L}}(X)\right)\right\}_{X}$, where $\rho_{\mathcal{L}}(X):=\bigcap_{\Lambda \in \mathcal{L}} \rho_{\Lambda}(X)$ for each $X$. Hence, the counterpart of Proposition 3.3 for $\Sigma$-VFCs can be written as follows.

Corollary 3.1. The $\Sigma$-VFCs $\mathbf{F R A} \mathbf{A}_{\mathcal{L}}^{c}$ form a $2^{n(\Sigma)}$-element Boolean sublattice in the lattice of all $\Sigma$-VFCs. In this sublattice the least element is $\left\{\left\{\nabla_{T_{\Sigma}(X)}\right\}\right\}_{X}$, the greatest element is $\left\{\left[\rho_{p a(\Sigma)}(X)\right)\right\}_{X}$, and for all $\mathcal{L}, \mathcal{M} \subseteq p a(\Sigma), \mathbf{F R A}_{\mathcal{L}}^{c} \vee \mathbf{F R A}_{\mathcal{M}}^{c}=$ $\left\{\left[\rho_{\mathcal{L} \cup \mathcal{M}}(X)\right)\right\}_{X}, \quad \mathbf{F R A}_{\mathcal{L}}^{c} \wedge \mathbf{F R A}_{\mathcal{M}}^{c}=\left\{\left[\rho_{\mathcal{L} \cap \mathcal{M}}(X)\right)\right\}_{X}$, and $\left(\mathbf{F R A}_{\mathcal{L}}^{c}\right)^{\mathcal{C}}=$ $\left\{\left[\rho_{p a(\Sigma) \backslash \mathcal{L}}(X)\right)\right\}_{X}$. The atoms of the sublattice are the $\Sigma-V F C s \mathbf{F P r o j}_{\Lambda}^{c}=$ $\left\{\left[\rho_{\Lambda}(X)\right)\right\}_{X}$ with $\Lambda \in p a(\Sigma)$.

In [11] it was shown that any rectangular algebra of finite type is isomorphic to the direct product of a finite family of projection algebras. In particular, any member of $\mathbf{F R A _ { \Sigma }}$ is isomorphic to the direct product of a finite family of finite projection algebras. By collecting together factors belonging to the same $\Sigma$-VFA FProj $_{\Lambda}$, this decomposition result can be expressed more precisely as follows.

Proposition 3.4. For any set of projection alphabets $\mathcal{L}=\left\{\Lambda_{1}, \ldots, \Lambda_{k}\right\} \subseteq p a(\Sigma)$, every algebra in $\mathbf{F R A _ { \mathcal { L } }}$ is isomorphic to a direct product $\mathcal{A}_{1} \times \cdots \times \mathcal{A}_{k}$ where $\mathcal{A}_{i} \in \mathbf{F P r o j}_{\Lambda_{i}}$ for $i=1, \ldots, k$.

\section{Projection and rectangular algebras as tree rec- ognizers}

We shall now consider the tree languages recognizable by projection algebras and rectangular algebras. For any $\Lambda \in p a(\Sigma)$ and any $\mathcal{L} \subseteq p a(\Sigma)$, let $F \operatorname{Proj}_{\Lambda}=$ $\left\{F \operatorname{Proj}_{\Lambda}(X)\right\}_{X}$ and $F R A_{\mathcal{L}}=\left\{F R A_{\mathcal{L}}(X)\right\}_{X}$ be the $\Sigma$-VTLs that correspond to FProj $_{\Lambda}$ and FRA $_{\mathcal{L}}$, respectively. The $\Sigma$-VTL $F R A_{p a(\Sigma)}$ may be denoted also by $F R A_{\Sigma}$.

It is easy to see that any $\Sigma X$-tree $t$ can be evaluated in a $\Lambda$-projection algebra $\mathcal{A}=(A, \Sigma)$ for an assignment $\alpha: X \rightarrow A$ simply by transporting the value $\alpha\left(\Lambda^{\bullet}(t)\right)$ 
along the path described by $\Lambda(t)$ from the leaf to the root. This is expressed formally by the following lemma.

Lemma 4.1. Let $\mathcal{A}=(A, \Sigma)$ be a $\Lambda$-projection algebra and let $\alpha: X \rightarrow A$ be any assignment. Then $t \alpha_{\mathcal{A}}=\alpha\left(\Lambda^{\bullet}(t)\right)$ for every $\Sigma X$-tree $t$.

Corollary 4.1. $T(\mathbf{A})=\left\{t \in T_{\Sigma}(X) \mid \alpha\left(\Lambda^{\bullet}(t)\right) \in F\right\}$ for any $\Sigma X$-recognizer $\mathbf{A}=(\mathcal{A}, \alpha, F)$ such that $\mathcal{A} \in \mathbf{F} \mathbf{P r o j}_{\Lambda}$.

For any $Y \subseteq X$, let $T_{\Lambda}(X, Y):=\left\{t \in T_{\Sigma}(X) \mid \Lambda^{\bullet}(t) \in Y\right\}$. By Proposition 3.2, the principal $\Sigma$-VFC $\left\{\left[\rho_{\Lambda}(X)\right)\right\}_{X}$ corresponds to the $\Sigma$-VFA FProj $_{\Lambda}$, and hence also to the $\Sigma$-VTL $F \operatorname{Proj}_{\Lambda}$. Clearly, the $\Sigma X$-tree languages saturated by $\rho_{\Lambda}(X)$ are exactly the sets $T_{\Lambda}(X, Y)$. By Remark 2.1 this fact yields the first part of the following proposition but we shall give a direct proof.

Proposition 4.1. For any projection alphabet $\Lambda \in p a(\Sigma)$ and any leaf alphabet $X$,

$$
\operatorname{FProj}_{\Lambda}(X)=\left\{T_{\Lambda}(X, Y) \mid Y \subseteq X\right\} .
$$

Moreover, a $\Sigma X$-tree language $T$ is in $F \operatorname{Proj}_{\Lambda}(X)$ if and only if $\mathrm{SA}(T)$ is either trivial or isomorphic to $\mathcal{P}_{\Lambda}$.

Proof. Firstly, $T(\mathbf{A})$ in Corollary 4.1 equals $T_{\Lambda}(X, Y)$ for $Y=\{x \in X \mid \alpha(x) \in F\}$. Conversely, for any given $T_{\Lambda}(X, Y)$, let $\mathbf{A}=\left(\mathcal{P}_{\Lambda}, \alpha, F\right)$ be the $\Sigma X$-recognizer where $\alpha(x)=1$ for $x \in Y, \alpha(x)=0$ for $x \in X \backslash Y$, and $F=\{1\}$. Then $T(\mathbf{A})=\{t \in$ $\left.T_{\Sigma}(X) \mid \alpha\left(\Lambda^{\bullet}(t)\right)=1\right\}=\left\{t \in T_{\Sigma}(X) \mid \Lambda^{\bullet}(t) \in Y\right\}=T_{\Lambda}(X, Y)$ by Corollary 4.1 and Lemma 4.1.

Let us now consider any tree language $T \subseteq T_{\Sigma}(X)$. If $\mathrm{SA}(T)$ is trivial or isomorphic to $\mathcal{P}_{\Lambda}$, then $T \in F \operatorname{Proj}_{\Lambda}(X)$ as both the trivial $\Sigma$-algebras and $\mathcal{P}_{\Lambda}$ are in $\operatorname{FProj}_{\Lambda}$. On the other hand, if $T \in \operatorname{FProj}_{\Lambda}(X)$, then $T$ is recognized by a $\Lambda$-projection algebra. By what we have shown above, this means that $T$ is of the form $T_{\Lambda}(X, Y)$ and therefore recognized by $\mathcal{P}_{\Lambda}$. If $\mathcal{P}_{\Lambda}$ is the minimal $\Sigma$-algebra recognizing $T$, then $\mathrm{SA}(T) \cong \mathcal{P}_{\Lambda}$, but otherwise $\mathrm{SA}(T)$ is trivial.

Every subdirectly irreducible algebra is syntactic, but the converse does not hold in general. However, Proposition 4.1 shows that here the two classes coincide.

Corollary 4.2. A projection algebra is syntactic if and only if it is subdirectly irreducible, i.e., iff it is trivial or isomorphic to one of the algebras $\mathcal{P}_{\Lambda}(\Lambda \in p a(\Sigma))$.

Although we know by the Variety Theorem 2.1 that $F \operatorname{Proj}_{\Lambda}$ is a $\Sigma$-VTL, it may be instructive to show also directly that, for any given projection alphabet $\Lambda \in p a(\Sigma)$, the sets $T_{\Lambda}(X, Y)$ form a $\Sigma$-VTL. Firstly, for any $X$ and $Y, Y^{\prime} \subseteq X$, we have $T_{\Lambda}(X, Y)^{\complement}=T_{\Lambda}(X, X \backslash Y)$ and $T_{\Lambda}(X, Y) \cup T_{\Lambda}\left(X, Y^{\prime}\right)=T_{\Lambda}\left(X, Y \cup \bar{Y}^{\prime}\right)$. Let us extend the function $\Lambda^{\bullet}$ to $\Sigma X$-contexts in the obvious way. Then we have for any $p \in C_{\Sigma}(X)$,

$$
p^{-1}\left(T_{\Lambda}(X, Y)\right)= \begin{cases}T_{\Sigma}(X)=T_{\Lambda}(X, X) & \text { if } \Lambda^{\bullet}(p) \in Y \\ \emptyset=T_{\Lambda}(X, \emptyset) & \text { if } \Lambda^{\bullet}(p) \in X \backslash Y \\ T_{\Lambda}(X, Y) & \text { if } \Lambda^{\bullet}(p)=\xi\end{cases}
$$


Finally, if $\varphi: \mathcal{T}_{\Sigma}(X) \rightarrow \mathcal{T}_{\Sigma}(Y)$ is a homomorphism and $Y^{\prime} \subseteq Y$, then $T_{\Lambda}\left(Y, Y^{\prime}\right) \varphi^{-1}=$ $T_{\Lambda}\left(X, X^{\prime}\right)$, where $X^{\prime}=\left\{x \in X \mid \Lambda^{\bullet}(x \varphi) \in Y^{\prime}\right\}$.

Following [15], we define the syntactic monoid congruence of a $\Sigma X$-tree language $T$ as the relation $\mu_{T}$ on $C_{\Sigma}(X)$ such that

$p \mu_{T} q \Leftrightarrow\left(\forall t \in T_{\Sigma}(X)\right)\left(\forall r \in C_{\Sigma}(X)\right)(t \cdot p \cdot r \in T \leftrightarrow t \cdot q \cdot r \in T) \quad\left(p, q \in C_{\Sigma}(X)\right)$,

and the syntactic monoid $\operatorname{SM}(T)$ of $T$ as the quotient monoid $C_{\Sigma}(X) / \mu_{T}$.

It is easy to see that if $T=T_{\Lambda}(X, Y)$ with $Y \neq X, \emptyset$, then $\mu_{T}$ has the congruence classes

(1) $[\xi]=\left\{p \in C_{\Sigma}(X) \mid \Lambda^{\bullet}(p)=\xi\right\}$,

(2) $\left[p_{+}\right]=\left\{p \in C_{\Sigma}(X) \mid \Lambda^{\bullet}(p) \in Y\right\}$, and

(3) $\left[p_{-}\right]=\left\{p \in C_{\Sigma}(X) \mid \Lambda^{\bullet}(p) \in X \backslash Y\right\}$.

Furthermore, $[\xi]$ is the identity element in $\operatorname{SM}(T)$ while $\left[p_{+}\right]$and $\left[p_{-}\right]$both are right zeros as $\left(p \cdot p_{+}, p_{+}\right),\left(p \cdot p_{-}, p_{-}\right) \in \mu_{T}$ for any $p_{+} \in\left[p_{+}\right], p_{-} \in\left[p_{-}\right]$and $p \in C_{\Sigma}(X)$. Hence the following corollary of Proposition 4.1.

Corollary 4.3. For any $\Lambda \in p a(\Sigma)$, any leaf alphabet $X$, and any $T \in F \operatorname{Proj}_{\Lambda}(X)$, the syntactic monoid $\operatorname{SM}(T)$ is either trivial or isomorphic to the 3-element monoid $M=\{1, a, b\}$ in which 1 is the identity element and the elements $a$ and $b$ are right zeros.

However, Corollary 4.3 does not mean that the $\Sigma$-VTL $F$ Proj $_{\Lambda}$ can be characterized by syntactic monoids. Indeed, the same syntactic monoids are obtained for every $\Lambda \in p a(\Sigma)$ and there are also completely different tree languages with syntactic monoids isomorphic to $M$.

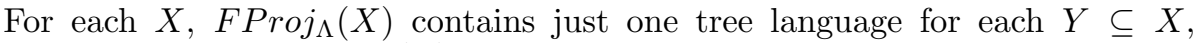
and hence $F \operatorname{Proj}_{\Lambda}(X)$ has $2^{|X|}$ elements. Let us consider the more general $\Sigma$ VTLs $F R A_{\mathcal{L}}(\mathcal{L} \subseteq p a(\Sigma))$, i.e., the joins of the $\Sigma$-VTLs $F \operatorname{Proj}_{\Lambda}$. By the Variety Theorem, Propositions 3.3 and 3.1 translate into the following proposition about $\Sigma$-VTLs.

Proposition 4.2. The $\Sigma$-VTLs $F R A_{\mathcal{L}}(\mathcal{L} \subseteq$ pa $(\Sigma))$ form a $2^{n(\Sigma)}$-element Boolean sublattice of the lattice of all $\Sigma$-VTLs. In this sublattice the least element is Triv $v_{\Sigma}=$ $\left\{\left\{\emptyset, T_{\Sigma}(X)\right\}\right\}_{X}$, the greatest element is $F R A_{\Sigma}$, and for all $\mathcal{L}, \mathcal{M} \subseteq p a(\Sigma), F R A_{\mathcal{L}} \vee$ $F R A_{\mathcal{M}}=F R A_{\mathcal{L} \cup \mathcal{M}}, F R A_{\mathcal{L}} \wedge F R A_{\mathcal{M}}=F R A_{\mathcal{L} \cap \mathcal{M}}$, and $F R A_{\mathcal{L}}^{\bar{\complement}}=F R A_{p a(\Sigma) \backslash \mathcal{L}}$. The atoms of the sublattice, the $\Sigma$-VTLs $\operatorname{FProj}_{\Lambda}(\Lambda \in p a(\Sigma))$, are minimal $\Sigma$ VTLs.

From Propositions 3.3 it also follows that for any $\mathcal{L} \subseteq p a(\Sigma)$ and any $X$, the members of $F R A_{\mathcal{L}}(X)$ are precisely the $\Sigma X$-tree languages saturated by $\rho_{\mathcal{L}}(X)$. Now, it is easy to see that, quite generally, if $\theta_{1}, \ldots, \theta_{n}(n \geq 1)$ are equivalences on a set $U$, then the subsets of $U$ saturated by $\theta_{1} \cap \ldots \cap \theta_{n}$ are precisely the sets that can be represented as finite unions of intersections $C_{1} \cap \ldots \cap C_{n}$, where for each 
$i \in[n], C_{i}$ is a subset of $U$ saturated by $\theta_{i}$. Hence the following description of the $\Sigma$-VTLs $F R A_{\mathcal{L}}$ is obtained by using Proposition 4.1. It could also be expressed by saying that $F R A_{\mathcal{L}}$ is the ring closure of $\bigcup_{\Lambda \in \mathcal{L}} F \operatorname{Proj}_{\Lambda}$.

Proposition 4.3. For any set $\mathcal{L}=\left\{\Lambda_{1}, \ldots, \Lambda_{n}\right\} \subseteq$ pa $(\Sigma)$ of projection alphabets and any $X$, the members of $F R A_{\mathcal{L}}(X)$ are precisely the unions of finitely many intersections

$$
T_{\Lambda_{1}}\left(X, Y_{1}\right) \cap \ldots \cap T_{\Lambda_{n}}\left(X, Y_{n}\right),
$$

where $Y_{1}, \ldots, Y_{n} \subseteq X$.

The following decidability result is obvious by the finiteness of $\mathbf{F R} \mathbf{A}_{\mathcal{L}}(X)$, but it also follows from the fact that a finite $\Sigma$-algebra belongs to $\mathbf{F R \mathbf { A } _ { \mathcal { L } }}$ iff it is isomorphic to a subdirect product of algebras $\mathcal{P}_{\Lambda}$ with $\Lambda \in p a(\Sigma)$.

Proposition 4.4. For any $\mathcal{L} \subseteq p a(\Sigma)$, it can be decided whether a given regular $\Sigma X$-tree language belongs to $F R A_{\mathcal{L}}$.

\section{Comparisons with other varieties}

It is to be expected that our varieties have little in common with most of the varieties of finite algebras or varieties of tree languages considered in the literature. Firstly, they are too small to contain other nontrivial varieties. In particular, the $\Sigma$-VTLs $F R A_{\mathcal{L}}$ contain no nonempty finite sets. On the other hand, the sets $T_{\Lambda}(X, Y)(\emptyset \subset Y \subset X)$ are not defined by any local properties of their trees - as usually is the case. Let us make this incomparability explicit for a few $\Sigma$-VTLs. The precise definitions of these can be found in [12] or [13], for example, but the following informal descriptions should suffice here.

In the $\Sigma$-VTL $N i l_{\Sigma}=\left\{N i l_{\Sigma}(X)\right\}_{X}$, each set $N i l_{\Sigma}(X)$ consists of the finite and the co-finite $\Sigma X$-tree languages, and $N i l^{a}$ is the $\Sigma$-VFA Nil $\mathrm{N}_{\Sigma}$ of nilpotent (finite) $\Sigma$-algebras (defined in [4]).

A $\Sigma X$-tree language $T$ is definite if there is a $k \geq 0$ such that the membership of a $\Sigma X$-tree in $T$ depends only on the 'root segment' of $t$ of height $k-1$. Similarly, $T$ is reverse definite if there is a $k \geq 0$ such that whether or not $t \in T$ depends just on the subtrees of $t$ of height $<k$. (In both cases, $k=0$ means that no testing is needed and, accordingly, $T=\emptyset$ or $T=T_{\Sigma}(X)$.) By allowing combinations of these two types of tests, we get the generalized definite tree languages. The three $\Sigma$-VTLs obtained this way are denoted by $D e f_{\Sigma}, R D e f_{\Sigma}$ and $G D e f_{\Sigma}$, and the corresponding $\Sigma$-VFAs by $\operatorname{Def}_{\Sigma}, \mathbf{R D e f}_{\Sigma}$ and $\mathbf{G D e f}_{\Sigma}$, respectively.

A fork of $\Sigma X$-tree is a configuration of the form $f\left(d_{1}, \ldots, d_{m}\right)$, where $f \in \Sigma_{m}$, $m>0$ and $d_{1}, \ldots, d_{m} \in \Sigma \cup X$. A $\Sigma X$-tree language $T$ is local if whether a $\Sigma X$-tree $t$ belongs to $T$ is determined by the set of forks appearing in $t$ and its root label. The $\Sigma$-VTL $L o c_{\Sigma}$ of locally testable $\Sigma$-tree languages is obtained as the Boolean closure of the $\Sigma$-family of local tree languages. Let $\mathbf{L o c}_{\Sigma}$ be the corresponding $\Sigma$-VFA.

Proposition 5.1. For any set of path alphabets $\mathcal{L} \subseteq p a(\Sigma)$, 
(a) $F R A_{\mathcal{L}} \cap \mathcal{V}=\operatorname{Triv}_{\Sigma}$ if $\mathcal{V}$ is $N i l_{\Sigma}, D e f_{\Sigma}, R D e f_{\Sigma}, G D e f_{\Sigma}$ or $L o c_{\Sigma}$, and

(b) $\mathbf{F R A}_{\mathcal{L}} \cap \mathbf{K}=\operatorname{Triv}_{\Sigma}$ if $\mathbf{K}$ is $\mathbf{N i l}_{\Sigma}, \mathbf{D e f}_{\Sigma}, \mathbf{R D e f}_{\Sigma}, \mathbf{G D e f}_{\Sigma}$ or $\mathbf{L o c}_{\Sigma}$.

Proof. By the Variety Theorem, assertions (a) and (b) are equivalent. The case $\mathcal{L}=\emptyset$ being trivial, we assume that $\mathcal{L} \neq \emptyset$.

Let us first assume that $\mathcal{L}$ consists of a single path alphabet $\Lambda$. Since $F \operatorname{Proj}_{\Lambda}$ is a minimal $\Sigma$-VTL and the intersection of any $\Sigma$-VTLs is a $\Sigma$-VTL, statement (a) holds for $\mathcal{L}=\{\Lambda\}$ if $F \operatorname{Proj}_{\Lambda}$ is not contained in any of the $\Sigma$-VTLs $\mathcal{V}$. To show this, we consider any $T_{\Lambda}(X, Y) \in \operatorname{FProj}_{\Lambda}(X)$ such that $\emptyset \subset Y \subset X$. Since $T_{\Lambda}(X, Y)$ is neither finite nor co-finite, it is not in $N_{i l}(X)$. To show that $T_{\Lambda}(X, Y)$ is not definite, we select any $f \in \Sigma, y \in Y$ and $x \in X \backslash Y$, and define two sequences of $\Sigma X$-trees by setting (1) $s_{0}=y, t_{0}=x$, and (2) $s_{n+1}=f\left(s_{n}, \ldots, s_{n}\right)$ and $t_{n+1}=f\left(t_{n}, \ldots, t_{n}\right)$ for all $n \geq 0$. Then, for every $k \geq 0$, the trees $s_{k}$ and $t_{k}$ have the same root segment of height $k-1$, but $s_{k} \in T_{\Lambda}(X, Y)$ while $t_{k} \notin T_{\Lambda}(X, Y)$, and therefore $T_{\Lambda}(X, Y)$ is not definite. Similar arguments can be used in the remaining cases. Since all the sets $T_{\Lambda}(X, Y)$ are recognized by $\mathcal{P}_{\Lambda}$, it follows that $\mathcal{P}_{\Lambda}$ cannot belong to any of the $\Sigma$-VFAs $\mathbf{N i l}_{\Sigma}, \mathbf{D e f}_{\Sigma}, \mathbf{R D e f}_{\Sigma}, \mathbf{G D e f}_{\Sigma}$ or $\mathbf{L o c}_{\Sigma}$.

Consider now the general case $\emptyset \neq \mathcal{L} \subseteq p a(\Sigma)$. Let $\mathbf{K}$ be any one of the $\Sigma$ -

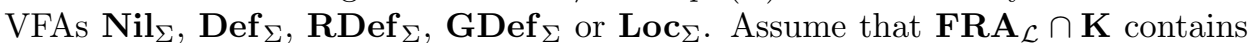
a nontrivial $\Sigma$-algebra $\mathcal{A}$. Then $\mathcal{A}$ would have a decomposition into a subdirect product of some subdirectly irreducible algebras $\mathcal{A}_{1}, \ldots, \mathcal{A}_{n}(n \geq 1)$ all of which

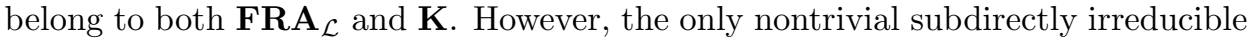
algebras in $\mathbf{F R A} \mathbf{A}_{\mathcal{L}}$ are the algebras $\mathcal{P}_{\Lambda}$ with $\Lambda \in \mathcal{L}$, and by the first part of the proof, these do not belong to $\mathbf{K}$. Therefore we must have $\mathbf{F R A} \mathbf{A}_{\mathcal{L}} \cap \mathbf{K}=\operatorname{Triv}_{\Sigma}$.

We conclude this section with two examples of $\Sigma$-VTLs that contain the $\Sigma$-VTLs $F R A_{\mathcal{L}}$. Thomas [15] calls a $\Sigma X$-tree language $T$ aperiodic if there is an $n \geq 0$ such that

$$
\left(\forall t \in T_{\Sigma}(X)\right)\left(\forall p, q \in C_{\Sigma}(X)\right)\left(t \cdot p^{n} \cdot q \in T \leftrightarrow t \cdot p^{n+1} \cdot q \in T\right) .
$$

The aperiodic $\Sigma$-tree languages form a $\Sigma$-VTL $A p_{\Sigma}$ that can be characterized by syntactic monoids [15].

Proposition 5.2. $F R A_{\Sigma} \subset A p_{\Sigma}$.

Proof. We begin by showing that $F \operatorname{Proj}_{\Lambda} \subset A p_{\Sigma}$ for every $\Lambda \in p a(\Sigma)$. Let $T=T_{\Lambda}(X, Y)$ be any set in $F \operatorname{Proj}_{\Lambda}(X)$. The remaining two cases being trivial, we may assume that $\emptyset \subset Y \subset X$. To show that for any $t \in T_{\Sigma}(X)$ and $p, q \in C_{\Sigma}(X)$, $t \cdot p \cdot q \in T$ iff $t \cdot p^{2} \cdot q \in T$ (our " $n$ " is 1 ), we distinguish two cases:

1. If $\Lambda^{\bullet}(q) \in X$, then $\Lambda^{\bullet}(t \cdot p \cdot q)=\Lambda^{\bullet}(q)=\Lambda^{\bullet}\left(t \cdot p^{2} \cdot q\right)$, and hence $t \cdot p \cdot q \in T$ iff $t \cdot p^{2} \cdot q \in T$.

2. If $\Lambda^{\bullet}(q)=\xi$, there are two subcases to consider. If $\Lambda^{\bullet}(p) \in X$, then $\Lambda^{\bullet}(t \cdot p$. $q)=\Lambda^{\bullet}(p)=\Lambda^{\bullet}\left(t \cdot p^{2} \cdot q\right)$, and hence $t \cdot p \cdot q \in T$ iff $t \cdot p^{2} \cdot q \in T$. If $\Lambda^{\bullet}(p)=\xi$, then $\Lambda^{\bullet}(t \cdot p \cdot q)=\Lambda^{\bullet}(t)=\Lambda \bullet\left(t \cdot p^{2} \cdot q\right)$, and again $t \cdot p \cdot q \in T$ iff $t \cdot p^{2} \cdot q \in T$. 
The inclusion $F R A_{\Sigma} \subseteq A p_{\Sigma}$ follows now because $F R A_{\Sigma}$ is the join of $\Sigma$-VTLs contained in the $\Sigma$-VTL $A p_{\Sigma}$. The inclusion is proper as all finite tree languages are aperiodic.

Finally, let us consider the tree languages recognized by deterministic tree recognizers that read their input trees starting at the root and accepting at the leaves. General treatments of this topic and further references can be found in [5], [6], and $[9]$.

A deterministic top-down $(D T) \Sigma$-algebra $\mathcal{B}=(B, \Sigma)$ consists of a non-empty set $B$ and a $\Sigma$-indexed family of top-down operations $f^{\mathcal{B}}: B \longrightarrow B^{m} \quad(m \in$ $\left.r(\Sigma), f \in \Sigma_{m}\right)$. Such a DT $\Sigma$-algebra $\mathcal{B}$ is finite if $B$ is a finite set. A $D T \Sigma X$ recognizer is a system $\mathbf{B}=\left(\mathcal{B}, b_{0}, \beta\right)$, where $\mathcal{B}=(B, \Sigma)$ is a finite DT $\Sigma$-algebra, the elements of which are called states, $b_{0} \in B$ is the initial state, and $\beta: X \rightarrow \wp(B)$ is the final state assignment. If we extend $\beta$ to a mapping $\beta_{\mathcal{B}}: T_{\Sigma}(X) \rightarrow \wp(B)$ by setting

(1) $\beta_{\mathcal{B}}(x)=\beta(x)$ for each $x \in X$, and

(2) $\beta_{\mathcal{B}}(t)=\left\{b \in B \mid f^{\mathcal{B}}(b) \in \beta_{\mathcal{B}}\left(t_{1}\right) \times \ldots \times \beta_{\mathcal{B}}\left(t_{m}\right)\right\}$ for $t=f\left(t_{1}, \ldots, t_{m}\right)$,

then for any $t \in T_{\Sigma}(X), \beta_{\mathcal{B}}(t)$ is the set of the states $b \in B$ such that $\mathbf{B}$ reaches every leaf of $t$ in an appropriate final state if started at the root of $t$ in state $b$. Accordingly, the tree language recognized by $\mathbf{B}$ is defined as $T(\mathbf{B}):=\left\{t \in T_{\Sigma}(X) \mid b_{0} \in \beta_{\mathcal{B}}(t)\right\}$. A $\Sigma X$-tree language $T$ is said to be DT-recognizable, if $T=T(\mathbf{B})$ for a DT $\Sigma X$ recognizer B. Let $D \operatorname{Rec}_{\Sigma}=\left\{D \operatorname{Rec}_{\Sigma}(X)\right\}_{X}$, where for each $X, D \operatorname{Rec}_{\Sigma}(X)$ is the set of all DT-recognizable $\Sigma X$-tree languages. ${ }^{1}$ It is well known that $D R e c_{\Sigma}$ is a proper subfamily of $R e c_{\Sigma}$.

Lemma 5.1. $F \operatorname{Proj}_{\Lambda} \subset \operatorname{DRec}_{\Sigma}$ for every $\Lambda \in p a(\Sigma)$.

Proof. If $T \in F \operatorname{Proj}_{\Lambda}(X)$, then $T=T(\mathbf{A})$ for a $\Sigma X$-recognizer $\mathbf{A}=\left(\mathcal{P}_{\Lambda}, \alpha, F\right)$, where $F=\{1\}$. We define a DT $\Sigma X$-recognizer $\mathbf{B}=(\mathcal{B}, 1, \beta)$ as follows. Let $\mathcal{B}=(\{0,1\}, \Sigma)$ be the DT $\Sigma$-algebra such that for any $m \in r(\Sigma), f \in \Sigma_{m}, f^{\mathcal{B}}(0)=$ $(0, \ldots, 0, \ldots, 0)$ and $f^{\mathcal{B}}(1)=(0, \ldots, 1, \ldots, 0)$, where the " 1 " is in position $i \in[m]$ if $f_{i} \in \Lambda$. For each $x \in X$, we set $\beta(x)=\{0, \alpha(x)\}$. By induction on $t \in T_{\Sigma}(X)$, it is easy to see that $\mathbf{B}$ reaches the leaf at the end of the path $\Lambda(t)$ in state 1 and all other leaves in state 0 . Hence, $\mathbf{B}$ accepts $t$ iff $\alpha\left(\Lambda^{\bullet}(t)\right)=1$, i.e., iff $t \in T(\mathbf{A})$.

The inclusion is proper since every singleton tree language is DR-recognizable.

Since $D R e c_{\Sigma}$ is not a $\Sigma$-VTL, Lemma 5.1 does not imply that $F R A_{\Sigma} \subseteq D R e c_{\Sigma}$. In fact, $F R A_{\mathcal{L}} \nsubseteq D R e c_{\Sigma}$ if $\mathcal{L}$ contains two distinct projection alphabets $\Lambda_{1}$ and $\Lambda_{2}$. Indeed, if $x$ and $y$ are two different symbols in $X$, then

$$
T:=\left\{t \in T_{\Sigma}(X) \mid \Lambda_{1}^{\bullet}(t)=x, \Lambda_{2}^{\bullet}(t)=y \text { or } \Lambda_{1}^{\bullet}(t)=y, \Lambda_{2}^{\bullet}(t)=x\right\}
$$

\footnotetext{
${ }^{1}$ Note that in the literature DT-recognizable tree languages are often called DR-recognizable tree languages (derived from "root-to-frontier" instead of the currently dominating "top-down").
} 
is recognized by $\mathcal{P}_{\Lambda_{1}} \times \mathcal{P}_{\Lambda_{2}}$ although it is not DT-recognizable. On the other hand, as shown by Jurvanen $[8,9]$, the Boolean closure $\mathfrak{B D R e c} c_{\Sigma}$ is the $\Sigma$-VTL generated by $D R e c_{\Sigma}$, and hence Lemma 5.1 yields the following conclusion. That the inclusion is proper, is easily seen by considering, for example, any set of the form $\{f(x, \ldots, x)\}$.

Proposition 5.3. $F R A_{\Sigma} \subset \mathfrak{B} D R e c_{\Sigma}$.

\section{$6 \quad$ Solidity properties}

Pöschel and Reichel [11] have shown that the rectangular $\Sigma$-algebras form the least nontrivial solid variety of $\Sigma$-algebras. For the general theory of solid varieties the reader may consult [10], for example. We shall consider the solidity properties of our $\Sigma$-VFAs and $\Sigma$-VTLs.

Let $\Xi:=\left\{\xi_{1}, \xi_{2}, \xi_{3}, \ldots\right\}$ be a set of variables which do not appear in any of the other alphabets. For any $n \geq 1$, let $\Xi_{n}:=\left\{\xi_{1}, \ldots, \xi_{n}\right\}$ and $T_{\Sigma}\left(\Xi_{n}\right)$ be the set of $n$-ary $\Sigma$-terms, and $T_{\Sigma}(\Xi):=\bigcup_{n \geq 1} T_{\Sigma}\left(\Xi_{n}\right)$ be the set of all $\Sigma$-terms with variables. If $t \in T_{\Sigma}\left(\Xi_{n}\right)$ and $t_{1}, \ldots, t_{n}$ are terms of any kind, then $t\left[t_{1}, \ldots, t_{n}\right]$ denotes the term obtained from $t$ by substituting for every occurrence of a variable $\xi_{1}, \ldots, \xi_{n}$ the respective term $t_{1}, \ldots, t_{n}$. The term function $A^{n} \rightarrow A$ defined by an $n$-ary term $t \in T_{\Sigma}\left(\Xi_{n}\right)$ in a $\Sigma$-algebra $\mathcal{A}=(A, \Sigma)$ is denoted by $t^{\mathcal{A}}$.

A hypersubstitution of type $\Sigma$ is a mapping $\varkappa: \Sigma \rightarrow T_{\Sigma}(\Xi)$ such that if $f \in \Sigma_{m}$, then $\varkappa(f) \in T_{\Sigma}\left(\Xi_{m}\right)$. Let $\mathcal{S}$ denote the set of all hypersubstitutions of type $\Sigma$. Any $\varkappa \in \mathcal{S}$ is extended to a mapping $\hat{\varkappa}: T_{\Sigma}(\Xi) \rightarrow T_{\Sigma}(\Xi)$ by setting $\hat{\varkappa}\left(\xi_{i}\right)=\xi_{i}$ for every $i \geq 1$, and $\hat{\varkappa}(t)=\varkappa(f)\left[\hat{\varkappa}\left(t_{1}\right), \ldots, \hat{\varkappa}\left(t_{m}\right)\right]$ for $t=f\left(t_{1}, \ldots, t_{m}\right)$. We let $\varkappa$ denote $\widehat{\varkappa}$, too.

For any $\varkappa \in \mathcal{S}$ and any $\Sigma$-algebra $\mathcal{A}=(A, \Sigma)$, the $\Sigma$-algebra $\varkappa(\mathcal{A})=(A, \Sigma)$ such that $f^{\varkappa(\mathcal{A})}=\varkappa(f)^{\mathcal{A}}$ for each $f \in \Sigma$, is a derived algebra of $\mathcal{A}$. In [7] it was noted that the solidity of varieties can be defined in terms of derived algebras, and the idea of solidity with respect to submonoids of the monoid of all hypersubstitutions of a given type was introduced in [2]. For a class $\mathcal{H} \subseteq \mathcal{S}$ of hypersubstitutions, a class $\mathbf{K}$ of $\Sigma$-algebras is said to be $\mathcal{H}$-solid if $\varkappa(\mathcal{A}) \in \mathbf{K}$ whenever $\mathcal{A} \in \mathbf{K}$ and $\varkappa \in \mathcal{H}$, and it is solid if it is $\mathcal{S}$-solid.

The first part of the following lemma can easily be verified by term induction. The second statement follows from the well-known fact that homomorphisms preserve also term functions.

Lemma 6.1. Let $\varkappa$ be a hypersubstitution of type $\Sigma$, and let $\mathcal{A}$ and $\mathcal{B}$ be any ¿-algebras.

(a) $t^{\varkappa(\mathcal{A})}=\varkappa(t)^{\mathcal{A}}$ for any $n \geq 1$ and any $n$-ary term $t \in T_{\Sigma}\left(\Xi_{n}\right)$.

(b) Any homomorphism $\varphi: \mathcal{A} \rightarrow \mathcal{B}$ is also a homomorphism from $\varkappa(\mathcal{A})$ to $\varkappa(\mathcal{B})$.

Let us call $\varkappa \in \mathcal{S}$ permutative if for all $m \in r(\Sigma)$ and $f \in \Sigma_{m}, \varkappa(f)=$ $g\left(\xi_{i_{1}}, \ldots, \xi_{i_{m}}\right)$ for some $g \in \Sigma_{m}$ and some permutation $\left(i_{1}, \ldots, i_{m}\right)$ of $(1, \ldots, m)$. 
Let $p \mathcal{S}$ denote the set of all these hypersubstitutions. In the terminology of [14], they are precisely the linear, nondeleting, symbol-to-symbol $\Sigma \Sigma$-substitutions.

Proposition 6.1. The $\Sigma-V F A s$ Triv $_{\Sigma}$ and $\mathbf{F R} \mathbf{A}_{\Sigma}$ are solid, and $\mathbf{F R} \mathbf{A}_{\Sigma}$ is the least nontrivial solid $\Sigma$-VFA. On the other hand, if $\emptyset \subset \mathcal{L} \subset p a(\Sigma)$, then $\mathbf{F R} \mathbf{A}_{\mathcal{L}}$ is not even $p \mathcal{S}$-solid.

Proof. Since the variety of rectangular $\Sigma$-algebras is solid by Theorem 5.1 of [11], also the $\Sigma$-VFA $\mathbf{F R A}_{\Sigma}$ is solid. Moreover, $\mathbf{F R A}_{\Sigma} \subseteq \mathbf{K}$ for every nontrivial solid $\Sigma$-VFA $\mathbf{K}$. Indeed, if $\mathcal{A}=(A, \Sigma)$ is any nontrivial member of $\mathbf{K}$, we obtain for any given $\Lambda \in p a(\Sigma)$ the $\Lambda$-projection algebra $\mathcal{B}=(A, \Sigma)$ as the derived algebra $\varkappa(\mathcal{A})$, if for any $m \in r(\Sigma)$ and $f \in \Sigma_{m}$, we set $\varkappa(f)=\xi_{i}$ for the $i \in[m]$ such that $f_{i} \in \Lambda$. From this it follows that $\mathbf{K}$ contains all the algebras $\mathcal{P}_{\Lambda}$ with $\Lambda \in p a(\Sigma)$, and hence all of $\mathbf{F R A} \mathbf{A}_{\Sigma}$.

Assume now that $\emptyset \subset \mathcal{L} \subset p a(\Sigma)$. To prove that $\mathbf{F R} \mathbf{A}_{\mathcal{L}}$ is not $p \mathcal{S}$-solid, it obviously suffices to show that for any two projection alphabets $\Lambda, \Lambda^{\prime} \in p a(\Sigma)$, there exists a permutative hypersubstitution $\varkappa$ for which $\mathcal{P}_{\Lambda^{\prime}}=\varkappa\left(\mathcal{P}_{\Lambda}\right)$. To define such a $\varkappa$, consider any $m \in r(\Sigma)$ and $f \in \Sigma_{m}$. If $f_{i} \in \Lambda$ and $f_{j} \in \Lambda^{\prime}(i, j \in[m])$, then we set $\varkappa(f)=f\left(\xi_{i_{1}}, \ldots, \xi_{i_{m}}\right)$, where $\left(i_{1}, \ldots, i_{m}\right)$ is the permutation of $(1, \ldots, m)$ that just transposes $i$ and $j$. It is then clear that $f^{\varkappa\left(\mathcal{P}_{\Lambda}\right)}=f^{\mathcal{P}_{\Lambda^{\prime}}}$.

To define the solidity of $\Sigma$-VTLs, we adapt some notions from [14] to the present setting of a fixed ranked alphabet. Firstly, if $\mathcal{H} \subseteq \mathcal{S}$ is a class of hypersubstitutions of type $\Sigma$, an $\mathcal{H}$-morphism $\varphi: T_{\Sigma}(X) \rightarrow T_{\Sigma}(Y)$ is defined by its underlying hypersubstitution $\varphi_{*} \in \mathcal{H}$ and a mapping $\varphi_{X}: X \rightarrow T_{\Sigma}(Y)$ as follows:

(1) $x \varphi=\varphi_{X}(x)$ for $x \in X$;

(2) $t \varphi=\varphi_{*}(f)\left[t_{1} \varphi, \ldots, t_{m} \varphi\right]$ for $t=f\left(t_{1}, \ldots, t_{m}\right)$.

The following fact is easily verified.

Lemma 6.2. If $\varphi: T_{\Sigma}(X) \rightarrow T_{\Sigma}(Y)$ is an $\mathcal{S}$-morphism, then $\varphi: \mathcal{T}_{\Sigma}(X) \rightarrow$ $\varphi_{*}\left(\mathcal{T}_{\Sigma}(Y)\right)$ is a homomorphism of $\Sigma$-algebras.

For any $\mathcal{H} \subseteq \mathcal{S}$, a $\Sigma$-VTL $\mathcal{V}=\{\mathcal{V}(X)\}_{X}$ is $\mathcal{H}$-solid if $T \in \mathcal{V}(Y)$ implies that $T \varphi^{-1} \in \mathcal{V}(X)$ for every $\mathcal{H}$-morphism $\varphi: T_{\Sigma}(X) \rightarrow T_{\Sigma}(Y)$. In particular, $\mathcal{V}$ is solid if it is $\mathcal{S}$-solid.

In [14] it was shown that any general variety of finite algebras and the corresponding general variety of tree languages (the "general" signifies that the ranked alphabet is not fixed) have matching solidity properties. Although restricting such general varieties to one given ranked alphabet does not yield exactly our $\Sigma$-VFAs and $\Sigma$-VTLs, this holds also here.

Lemma 6.3. Let $\mathcal{H}$ be any class of hypersubstitutions of type $\Sigma$. If a $\Sigma$-VFA $\mathbf{K}$ is $\mathcal{H}$-solid, then so is the $\Sigma$-VTL $\mathbf{K}^{t}$.

Proof. Consider any $\mathcal{H}$-morphism $\varphi: T_{\Sigma}(X) \rightarrow T_{\Sigma}(Y)$. If $T \in \mathbf{K}^{t}(Y)$, then there exist an algebra $\mathcal{A}=(A, \Sigma)$ in $\mathbf{K}$ and a homomorphism $\psi: \mathcal{T}_{\Sigma}(Y) \rightarrow \mathcal{A}$ such that 
$T=F \psi^{-1}$ for some $F \subseteq A$. By Lemmas 6.2 and $6.1, \varphi: \mathcal{T}_{\Sigma}(X) \rightarrow \varphi_{*}\left(\mathcal{T}_{\Sigma}(Y)\right)$ and $\psi: \varphi_{*}\left(\mathcal{T}_{\Sigma}(Y)\right) \rightarrow \varphi_{*}(\mathcal{A})$ are homomorphisms. Hence $\varphi \psi: \mathcal{T}_{\Sigma}(X) \rightarrow \varphi_{*}(\mathcal{A})$ is a homomorphism and $T \varphi^{-1}=F(\varphi \psi)^{-1}$. Since $\varphi_{*}(\mathcal{A}) \in \mathbf{K}$, this means that $T \varphi^{-1} \in \mathbf{K}^{t}(X)$.

Proving the converse of Lemma 6.3 would require some further preparations, so we avoid its use and just refer the reader to [14] for the corresponding fact about general varieties.

Proposition 6.2. The $\Sigma-V T L s$ Triv $v_{\Sigma}$ and $F R A_{\Sigma}$ are solid, and $F R A_{\Sigma}$ is the least nontrivial $\Sigma$-VTL. However, if $\emptyset \subset \mathcal{L} \subset p a(\Sigma)$, then $F R A_{\mathcal{L}}$ is not even $p \mathcal{S}$-solid.

Proof. That Triv $v_{\Sigma}$ and $F R A_{\Sigma}$ are solid follows from Proposition 6.1 by Lemma 6.3 .

To show that $F R A_{\mathcal{L}}$ is not $p \mathcal{S}$-solid for $\emptyset \subset \mathcal{L} \subset p a(\Sigma)$, consider any $\Lambda, \Lambda^{\prime} \in$ $p a(\Sigma)$ such that $\Lambda \in \mathcal{L}$, but $\Lambda^{\prime} \notin \mathcal{L}$. Let $X=\{x, y\}$ and $\varphi: T_{\Sigma}(X) \rightarrow T_{\Sigma}(X)$ be the $p \mathcal{S}$-morphism such that $\varphi_{*}$ is the hypersubstitution $\varkappa$ defined in the second part of the proof of Proposition 6.1, and $\varphi_{X}(x)=x$ and $\varphi_{X}(y)=y$. Then $T_{\Lambda}(X,\{y\}) \in$ $F R A_{\mathcal{L}}(X)$, but $T_{\Lambda}(X,\{y\}) \varphi^{-1}=T_{\Lambda^{\prime}}(X,\{y\}) \notin F R A_{\mathcal{L}}(X)$.

\section{References}

[1] S. Burris and H.P. Sankappanavar, A Course in Universal Algebra. SpringerVerlag, New York 1981.

[2] K. Denecke and M. Reichel, Monoids of hypersubstitutions and M-solid varieties. In: Contributions to General Algebra 9 (ed. G. Pilz), Hölder-PichlerTempsky, Wien-Stuttgart 1995, 117-126.

[3] Z. Ésik, A variety theorem for trees and theories, Publicationes Mathematicae Debrecen 54 Supplementum (1999), 711-762.

[4] F. Gécseg and B. Imreh, On a special class of automata. In: Automata, Languages and Programming Systems, Proc. Conf. Salgótárjan May 23-26, 1988 (eds. F. Gécseg and I. Peák), Department of Mathematics, Karl Marx University of Economics, Budapest 1988, 141-152.

[5] F. Gécseg and M. Steinby, Tree Automata, Akadémiai Kiadó, Budapest 1984.

[6] F. Gécseg and M. Steinby, Tree languages. In: Handbook of Formal Languages, Vol. 3 (eds. G. Rozenberg and A. Salomaa), Springer-Verlag, Berlin 1997, 169.

[7] E. Graczyńska and D. Schweigert, Hypervarieties of a given type, Algebra Universalis 27 (1990), 303-318.

[8] E. Jurvanen, The Boolean closure of DR-recognizable tree languages, Acta Cybernetica 10 (1992), 255-272.

[9] E. Jurvanen, On tree languages defined by deterministic root-to-frontier recognizers, PhD thesis, Department of Mathematics, University of Turku, Turku 1995.

[10] J. Koppitz and K. Denecke, M-Solid Varieties of Algebras, Springer Science+Business Media, New York 2006. 
[11] R. Pöschel and M. Reichel, Projection algebras and rectangular algebras. In: General Algebra and Applications (eds. K. Denecke and H. -J. Vogel), Heldermann Verlag, Berlin 1993, 180-194.

[12] M. Steinby: A theory of tree language varieties. In: Tree Automata and Languages (eds. M. Nivat and A. Podelski), North-Holland, Amsterdam 1992, 57-81.

[13] M. Steinby, Algebraic classifications of regular tree languages. In: Structural Theory of Automata, Semigroups, and Universal Algebra (eds. V.B. Kudryavtsev and I.G. Rosenberg), Springer, Dordrecht 2005, 381-432.

[14] M. Steinby, On the solidity of general varieties of tree languages, Discussiones Mathematicae. General Algebra and Applications 32 (2012), 23-53.

[15] W. Thomas, Logical aspects in the study of tree languages. In: 9th Colloquium on Trees in Algebra and Programming (Proc. 9th CAAP, Bordeaux 1984, ed. B. Courcelle), Cambridge University Press, London 1984, 31-49.

Received 14th June 2015 